\title{
A Japanese Patient with Gaucher Disease Treated with the Oral Drug Eliglustat as Substrate Reducing Therapy
}

\author{
Naoto Komada ${ }^{a}$ Toshinari Fujiwara ${ }^{a}$ Hideyuki Yoshizumi ${ }^{a}$ Hiroyuki Idab \\ Kazuya Shimodac \\ aNational Hospital Organization Miyakonojou Medical Center, Miyazaki, Japan; \\ ${ }^{b}$ Department of Pediatrics, Jikei University School of Medicine, Tokyo, Japan; 'Department \\ of Gastroenterology and Hematology, Faculty of Medicine, University of Miyazaki, Miyazaki, \\ Japan
}

\section{Keywords}

Enzyme replacement therapy - Gaucher disease - Splenomegaly - Substrate reducing therapy . Thrombocytopenia

\begin{abstract}
Gaucher disease is a rare genetic disorder caused by the deficiency of acid $\beta$-glucosidase to effectively catalyze the degradation of glucosylceramide to glucose and ceramide. We report here the case of a 31-year-old male Japanese patient with Gaucher disease who switched from enzyme replacement therapy (ERT) to substrate reducing therapy (SRT). Liver dysfunction was identified at a routine medical checkup, and the patient was referred to our hospital with "idiopathic liver disease." Clinical laboratory tests indicated thrombocytopenia and splenomegaly, which are characteristic symptoms of Gaucher disease. To definitively diagnose Gaucher disease, a bone marrow biopsy and acid $\beta$-glucosidase activity measurement were conducted; the results supported a diagnosis of Gaucher disease. This case emphasizes that it is possible for periodic medical checkups in adults to lead to the diagnosis of rare genetic disorders. The patient underwent ERT treatment with imiglucerase for 5 years; the platelet count rapidly increased and the spleen size rapidly decreased, indicating a good response to the drug. However, the patient increasingly felt the burden of visiting the hospital for $2 \mathrm{~h}$ of infusion ERT every 2 weeks. Consequently, it was jointly decided that he should switch from ERT to SRT with an oral drug. This switch was successful with no deterioration of laboratory data. This case report is the first to describe a Japanese Gaucher disease patient treated with eliglustat for $>2$ years. We showed that SRT is a well-tolerated and effective option for the treatment of Gaucher disease.
\end{abstract}




\section{Introduction}

Gaucher disease (GD) is an autosomal recessive lysosomal storage disorder resulting from a deficiency in the gene encoding acid $\beta$-glucosidase (GBA), which catalyzes the hydrolysis of glucosylceramide (GL-1) to glucose and ceramide [1]. Gaucher first described finding abnormal cells in an enlarged spleen in 1882 [2]. Further reports have demonstrated that GD is a multisystem disorder predominantly affecting reticuloendothelial cells. The resultant lysosomal accumulation of GL-1 throughout the body leads to progressive and debilitating clinical manifestations, including anemia, thrombocytopenia, hepatosplenomegaly, bone pain, and sometimes neurological disorders [3]. The 3 subtypes of GD are classified by the presence and extent of central nervous system (CNS) symptoms: type $1 \mathrm{GD}$, no CNS manifestations; type $2 \mathrm{GD}$, acute and severe CNS manifestations soon after the birth; and type $3 \mathrm{GD}$, a mixture of type 1 and type $3 \mathrm{GD}$, chronic and mild CNS manifestations.

GD is a very rare inborn error of metabolism in Japan; indeed, only approximately 150 cases have been reported. Diagnosis of GD at older age is also relatively rare, and the oldest Japanese patient is 69 years old [4]. GD is often considered a differential diagnosis when both thrombocytopenia and splenomegaly are found in relatively young patients. Therefore, if adult patients present with typical GD-related symptoms, most physicians do not suspect GD, but possibly diagnose idiopathic liver diseases or blood disorders as differential diagnoses. The current case concerns an adult patient with thrombocytopenia and splenomegaly diagnosed with GD. On periodic medical checkups, the patient was identified as having liver dysfunction. However, no specific disease was diagnosed, and the patient was considered to be suffering from idiopathic liver disease.

A definitive diagnosis of GD is determined by the low enzymatic activity of GBA in total leucocytes or cultured fibroblasts. Moreover, bone marrow examination is often performed to confirm the existence of Gaucher cells. Gaucher cells are macrophages with accumulated GBA substrate GL-1. The GD phenotypic speculation is usually conducted with reference to mutation information in previous reports or databases such as the Human Gene Mutation Database $\left(\right.$ HGMD $\left.^{\circledR}\right)$.

There are several treatment options for GD patients. These include enzyme replacement therapy (ERT), substrate reducing therapy (SRT), pharmacological chaperone therapy, and allogeneic hematopoietic transplantation. In Japan, once GBA deficiency is determined, ERT, recombinant GBA such as imiglucerase [5] and velaglucerase alfa [6], is the standard of care. Recently, oral SRT has been developed. This therapy inhibits glucosylceramide synthase and consequently reduces the production of GL-1. In Japan, only 1 SRT drug, eliglustat, is commercially available since its approval in 2015. Patients to be treated with eliglustat undergo CYP2D6 genotype testing to determine whether they are extensive, intermediate, or poor metabolizers, and the dosage is adjusted accordingly. Treatment with eliglustat has become more common, but there are no case studies of Japanese GD patients treated with eliglustat for a long time. Consequently, this report is the first covering a GD patient switching from ERT to SRT and undergoing treatment with eliglustat for $>2$ years in Japan.

\section{Case Report}

The patient was a 31-year-old Japanese man who had splenomegaly of unknown cause from birth. At a periodic medical examination in the early 2000s, he was found to have thrombocytopenia and liver dysfunction. No diagnosis or treatment was given, and the subject was

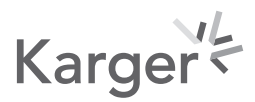


Table 1. Laboratory data obtained at the first medical examination at our hospital

\begin{tabular}{cllr}
\hline Peripheral blood & \multicolumn{3}{c}{$\begin{array}{c}\text { Differential white } \\
\text { blood count, } \%\end{array}$} \\
WBC (3.0-9.1) & $8.4 \times 10^{9} / \mathrm{L}$ & Neut & 39 \\
Hb (12.9-17.4) & $13.3 \mathrm{~g} / \mathrm{dL}$ & Ly & 55 \\
Ht (38.6-50.9) & $39.2 \%$ & Mo & 4 \\
Plt (143-333) & $105 \times 10^{9} / \mathrm{L}$ & Eo & 0 \\
& & Ba & 2 \\
Biochemical examination & & Hepatitis viral marker \\
T-Bil (0.2-1.2) & $2.0 \mathrm{mg} / \mathrm{dL}$ & HCV-Ab & $(-)$ \\
AST (8-38) & $80 \mathrm{IU} / \mathrm{L}$ & & \\
ALT (4-44) & $122 \mathrm{IU} / \mathrm{L}$ & & \\
ALP (104-338) & $708 \mathrm{mg} / \mathrm{dL}$ & & \\
$\gamma$-GTP (16-73) & $208 \mathrm{IU} / \mathrm{L}$ & & \\
CRP (<0.3) & $0.57 \mathrm{mg} / \mathrm{dL}$ & &
\end{tabular}

simply followed up for the following 3 years. Periodic medical examinations during this follow-up period indicated normal liver function. Nevertheless, 4 years after thrombocytopenia had been identified, the patient again experienced the symptoms of liver dysfunction and underwent detailed examination at another institution. However, no underlying cause was identified. The following year, the patient's liver function test results returned to normal, and follow-up was continued.

Six years after thrombocytopenia had first been identified, the patient visited a hospital complaining of low-grade fever for a few days. Basic hematologic tests again indicated thrombocytopenia and liver dysfunction, and the patient was referred to our hospital with "idiopathic liver disease." During the clinical course, the patient essentially appeared to be healthy. His family history indicated that his parents had not displayed any similar clinical symptoms.

\section{First Laboratory Data}

Laboratory examinations (shown in Table 1) revealed elevated levels of hepatobiliary enzymes: total bilirubin, $2.0 \mathrm{mg} / \mathrm{dL}$; aspartate transaminase, $80 \mathrm{IU} / \mathrm{L}$; alanine aminotransferase, $122 \mathrm{IU} / \mathrm{L}$; alkaline phosphatase, $708 \mathrm{mg} / \mathrm{dL}$; and $\gamma$-glutamyl transpeptidase, $208 \mathrm{IU} / \mathrm{L}$. Mild thrombocytopenia was also noted: platelet count, $105 \times 10^{9} / \mathrm{L}$. Hepatitis B and C viral markers were negative.

\section{Abdominal Ultrasonography}

On abdominal ultrasonography, splenomegaly and mild fatty liver were observed, but no cirrhotic changes (shown in Fig. 1a). We evaluated the size of the spleen by using the splenic index (SI). SI is calculated by multiplying the major axis by the minor axis by 0.9 . On the abdominal ultrasonography examination, the SI was 110.9 at the first examination.

\section{Bone Marrow Examination}

To rule out hematologic disease, we consulted a hematologist. Seven years after thrombocytopenia had first been identified, bone marrow aspiration and biopsy were performed. As a result, large macrophage-like cells having an eccentric nucleus and abundant cytoplasm 

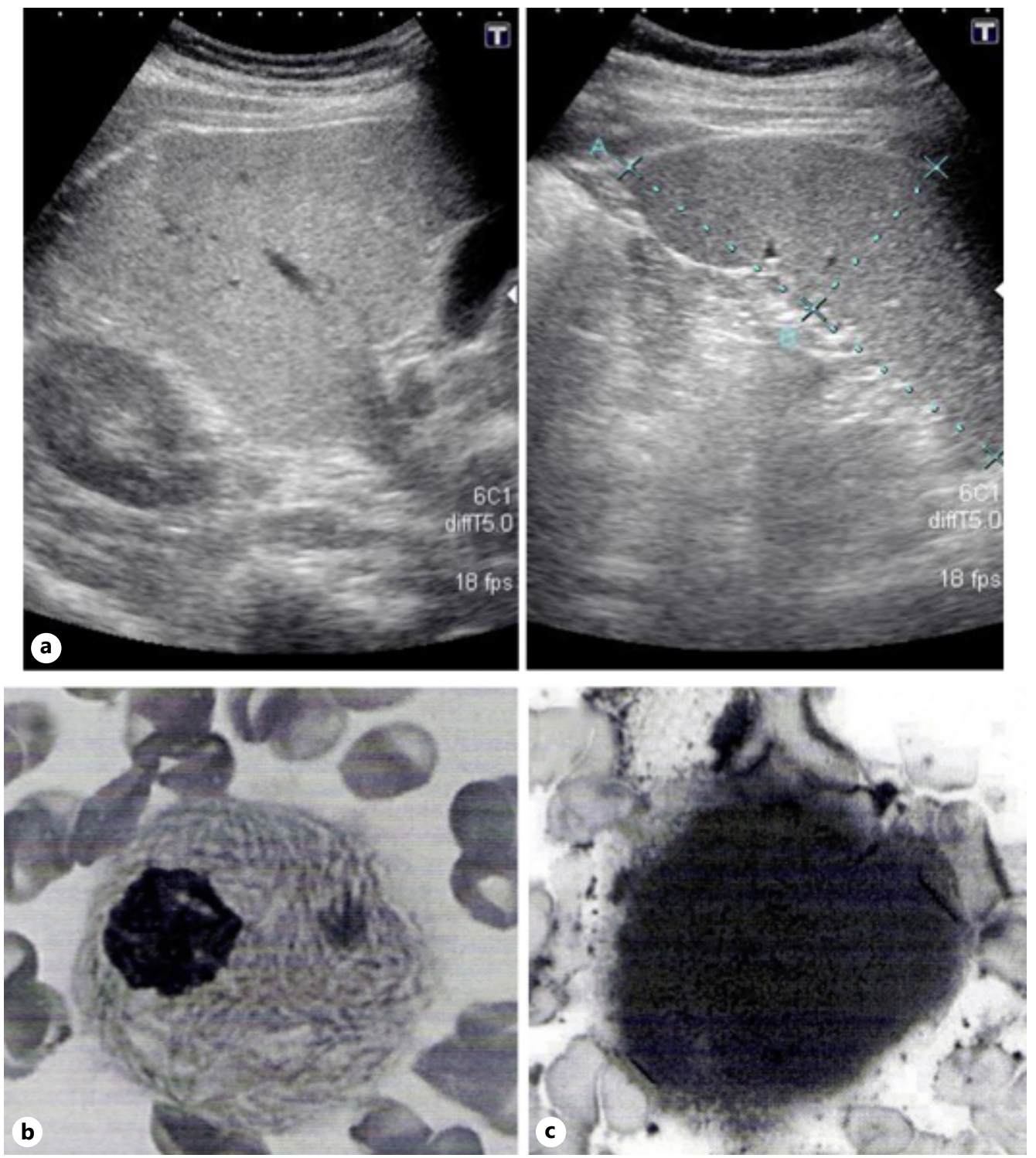

Fig. 1. Abdominal ultrasonography and bone marrow examination. a Splenomegaly and mild fatty change of the liver were noted, but no cirrhotic changes were evident. b Large cells with small, eccentrically located nuclei and cytoplasm with a characteristic wrinkled appearance were noted. c Histochemically, these large cells stained positive for acid phosphatase.

with a stratified appearance were observed. These findings were suggestive of the Gaucher cell phenotype. To confirm the diagnosis, a bone marrow examination was performed again at another institution. Large cells with small eccentrically located nuclei and cytoplasm with a characteristic wrinkled appearance (shown in Fig. 1b) were noted. Histochemically, these cells stained positive for acid phosphatase (shown in Fig. 1c). Consequently, these cells were identified as Gaucher cells.

\section{GBA Enzymatic Activity}

To obtain a definitive diagnosis, the patient's samples were sent to Professor Ida, Department of Pediatrics, Jikei University School of Medicine. The GBA enzymatic activity of 
Fig. 2. Time course of the platelet count and SI after the start of ERT. a The platelet count increased rapidly and was maintained over 5 years after imiglucerase infusion therapy was commenced. b Splenomegaly also rapidly improved. SI, splenic index; ERT, enzyme replacement therapy.

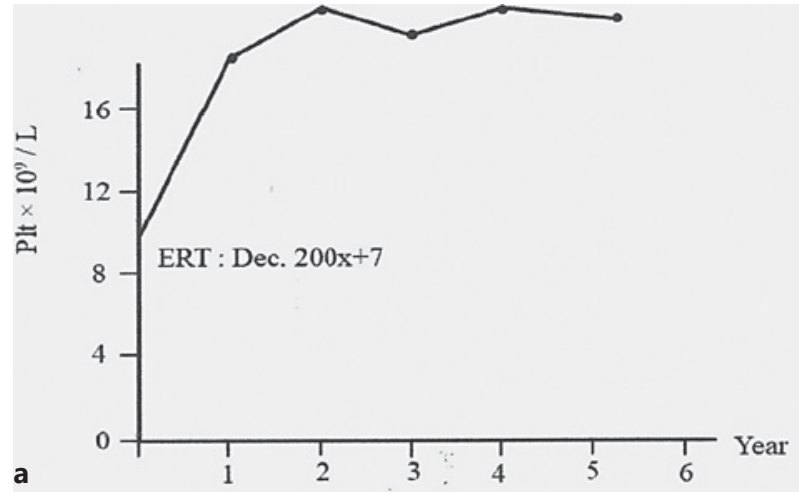

a

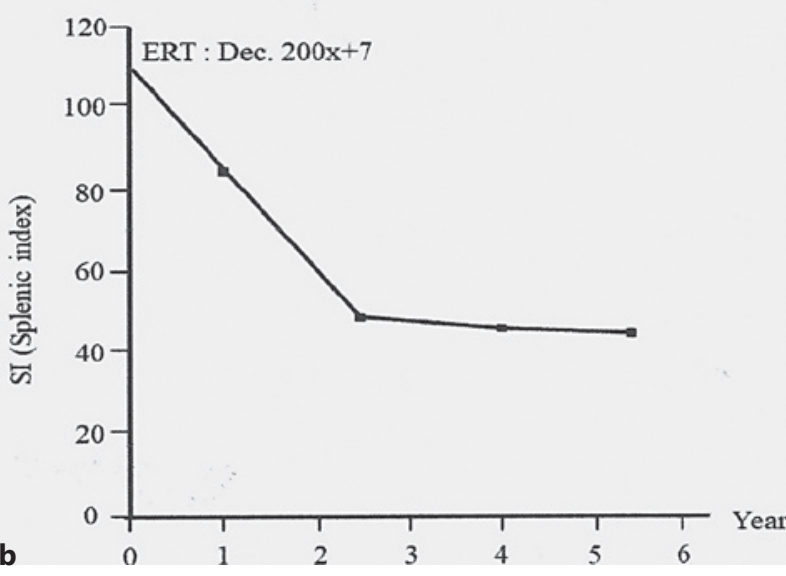

cultured fibroblasts was markedly reduced (38.6 nmol/h/mg), compared with normal controls (2,290.6-2,639.2 nmol/h/mg) and Gaucher controls (141.8 nmol/h/mg). As a result, the patient was diagnosed with Gaucher disease. Molecular genetic testing indicated that the patient was compound heterozygous for L444P and D409H mutations of GBA.

\section{Enzyme Replacement Therapy}

ERT was started once GD was diagnosed and consisted of infusion therapy of 60 units/ $\mathrm{kg}$ of imiglucerase every 2 weeks. After ERT was commenced, the platelet count increased rapidly. Initially, the patient's platelet count was $105 \times 10^{9} / \mathrm{L}$, but after ERT, the count increased to $140 \times 10^{9} / \mathrm{L}$ at 2 weeks and to $160 \times 10^{9} / \mathrm{L}$ at 1 month. After 2 months of ERT treatment, the platelet count was $220 \times 10^{9} / \mathrm{L}$. Furthermore, the patient's platelet count was successfully maintained within the normal range for the following 5 years without adverse events (shown in Fig. 2a). Splenomegaly also responded well to ERT and rapidly improved (shown in Fig. 2b).

\section{Substrate Reduction Therapy}

In Japan, eliglustat is commercially available as SRT for GD. The drug package insert states the requirement for CYP2D6 genotyping before prescribing eliglustat. This is because eliglustat is extensively metabolized by CYP2D6. Consequently, we investigated the patient's CYP2D6 phenotype and established that he was an extensive CYP2D6 metabolizer. The eliglustat dose for SRT for extensive metabolizers was $100 \mathrm{mg}$ twice daily. The patient was started on this SRT regimen; he was not taking any other medications or undergoing any 
Fig. 3. Time course of platelet count and SI during 5 years of ERT and 2 years of SRT. a The patient's platelet count remained stable at around $190 \times 10^{9} / \mathrm{L}$, and b splenomegaly continued to gradually improve after switching to SRT. SI, splenic index; ERT, enzyme replacement therapy; SRT, substrate reducing therapy.
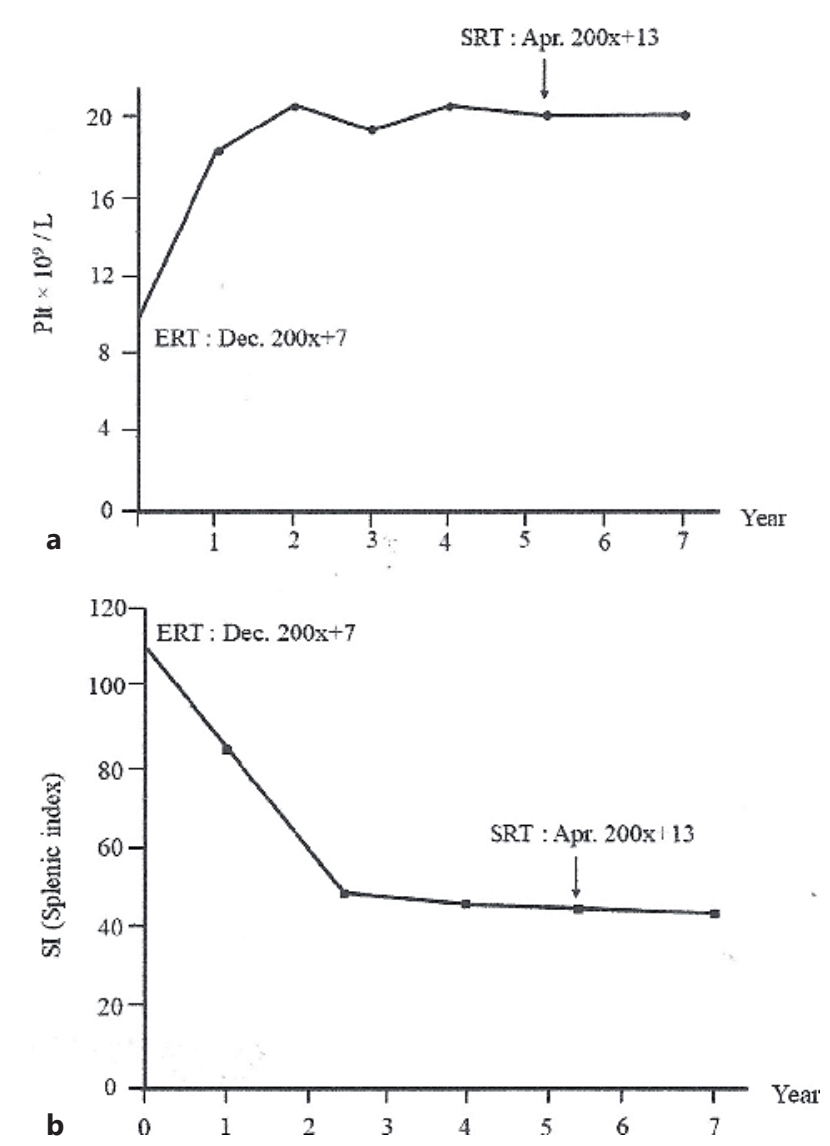

other therapies, and treatment with eliglustat did not cause any complications. On conversion from ERT to SRT with eliglustat, the platelet count remained stable at around $190 \times 10^{9} / \mathrm{L}$, and splenomegaly gradually improved (shown in Fig. 3).

\section{Discussion}

Patients with GD often experience long delays between the first indication of abnormalities, such as thrombocytopenia/splenomegaly, and diagnosis [7]. Although an enzymatic assay for GBA has been available since 1970 [8], tissue sampling, including bone biopsies, is also widely performed as the first step in the hematological diagnostic process [9]. In an Italian population presenting with splenomegaly and/or thrombocytopenia, the prevalence of GD was high at 3.6\% (95\% CI: 1.4-7.2) [10]. Actually, in Japan, a Gaucher disease patient was diagnosed by the identification of thrombocytopenia at a routine medical checkup. In our case, liver dysfunction was the first abnormal symptom, subsequently conducting diagnosis of Gaucher disease. Therefore, if a patient is found to have splenomegaly without cirrhosis, GD should actively be considered in the differential diagnosis.

In the present case, molecular genetic testing indicated the compound heterozygote mutations L444P and D409H, considered as type 3 GD [11], but the patient has no neurological symptoms at present. Some type $1 \mathrm{GD}$ patients have developed neurological symptoms 
during the clinical course of the disease [12]. Consequently, this patient should be observed carefully.

ERT, for which imiglucerase [5] and velaglucerase alfa [6] are widely used, usually results in marked improvements in symptoms in GD patients. In particular, visceral and hematological parameters improve [13]. In the current patient, during ERT, the platelet count increased and the spleen size decreased rapidly without any side effects. However, it is difficult to maintain good compliance for ERT because patients are required to attend hospital every 2 weeks to undergo infusion therapy that lasts $2 \mathrm{~h}$. Consequently, the current patient had to take a whole day off work. As a result, his quality of life was reduced, and he lost job opportunities and suffered a decreased income.

Eliglustat, a form of SRT, is as effective as ERT [14]. Consequently, worldwide regulatory agencies, including the FDA and EMA, have approved eliglustat as a first-line therapy for patients with GD [15]. Because eliglustat is an orally administered drug, patients need not visit hospitals every 2 weeks to undergo ERT. At present, this patient visits hospital to be monitored for GD-related symptoms and prescribed eliglustat only once every 3 months. In addition, he is not currently required to stay longer time at the hospital for the treatment of ERT, and thus he mentions he is able to continue his job without getting sick leaves after starting SRT treatment. Moreover, after switching from ERT to eliglustat, the current patient has remained stable for $>2$ years. In the current case, the effectiveness of eliglustat was confirmed, and eliglustat improved the patient's quality of life.

\section{Statement of Ethics}

All procedures were carried out in accordance with the ethical standards laid down in the 1964 Declaration of Helsinki and its later amendments. Written informed consent was obtained from the patient for publication of this case report and any accompanying images. This study protocol was reviewed, and the need for approval was waived by the Ethics Review Committee of the Miyakonojou Medical Center.

\section{Conflict of Interest Statement}

The authors of this manuscript do not have any conflicts of interest to declare.

\section{Funding Sources}

This manuscript did not receive any funding.

\section{Author Contributions}

All authors approved the final version of the manuscript.

\section{Data Availability Statement}

All data generated or analyzed during this study are included in this article. Further enquiries can be directed to the corresponding author. 


\section{References}

1 Brady RO, Kanfer JN, Shapiro D. Metabolism of glucocerebrosides. II. Evidence of an enzymatic deficiency in Gaucher's disease. Biochem Biophys Res Commun. 1965;18:221-5.

2 Gaucher PCE. De l'epithelioma primitive de la rate. Ref Type: Thesis/Dissertation, Faculte de Medicine de Paris; 1882

3 Charrow J, Andersson HC, Kaplan P, Kolodny EH, Mistry P, Pastores G, et al. The Gaucher registry: demographics and disease characteristics of 1698 patients with Gaucher disease. Arch Intern Med. 2000;160:283543.

4 Kawahara S, Kato A, Oshimi K, Ida H. [Gaucher disease type I diagnosed at 63 years old presenting with thrombocytopenia]. Rinsho Ketsueki. 2008;49:335-9.

5 Grabowski GA, Barton NW, Pastores G, Dambrosia JM, Banerjee TK, McKee MA, et al. Enzyme therapy in type 1 Gaucher disease : comparative efficacy of mannose-terminated glucocerebrosidase from natural and recombinant sources. Ann Intern Med. 1995;122(1):33-9.

6 Elstein D, Cohn GM, Wang N, Djordjevic M, Brutaru C, Zimran A. Early achievement and maintenance of the therapeutic goals using velaglucerase alfa in type 1 Gaucher disease. Blood Cells Mol Dis. 2011;46:119-23.

7 Mistry PK, Sadan S, Yang R, Yee J, Yang M. Consequences of diagnostic delays in type 1 Gaucher disease: the need for greater awareness among hematologists-oncologists and an opportunity for early diagnosis and intervention. Am J Hematol. 2007;82:697-701.

8 Beutler E, Kuhl W. The diagnosis of the adult type of Gaucher's disease and its carrier state by demonstration of deficiency of beta-glucosidase activity in peripheral blood leukocytes. J Lab Clin Med. 1970;76:747-55.

9 Giraldo P, Pocovi M, Perez-Calvo J, Rubio-Felix D, Giralt M. Report of the Spanish Gaucher's disease registry: clinical and genetic characteristics. Haematologica. 2000;85(8):792-9.

10 Motta I, Filocamo M, Poggiali E, Stroppiano M, Dragani A, Consonni D, et al. A multicentre observational study for early diagnosis of Gaucher disease in patients with splenomegaly and/or thrombocytopenia. Eur J Haematol. 2016 Apr;96(4):352-9.

11 Ida H, Rennert OM, Kawame H, Maekawa K, Eto Y. Mutation prevalence among 47 unrelated Japanese patients with Gaucher disease: identification of four novel mutations. J Inherit Metab Dis. 1997;20:67-73.

12 Tajima A, Yokoi T, Ariga M, Ito T, Kaneshiro E, Eto Y, et al. Clinical and genetic study of Japanese patients with type 3 Gaucher disease. Mol Genet Metab. 2009;97(4):272-7.

13 Weinreb NJ, Charrow J, Andersson HC, Kaplan P, Kolodny EH, Mistry P, et al. Effectiveness of enzyme replacement therapy in 1028 patients with type 1 Gaucher disease after 2 to 5 years of treatment: a report from the Gaucher registry. Am J Med. 2002;113:112-9.

14 Cox TM, Drelichman G, Cravo R, Balwari M, Burrow TA, Martins AM, et al. Eliglustat compared with imiglucerase in patients with Gaucher's disease type 1 stabilised on enxyme replacement therapy: a phase 3, randomized, open-label, non-inferiority trial. Lancet. 2015;385:2355-62. (Erratum in: Lancet. 2015 Jun 13; 385:2354)

15 Poole RM. Eliglustat: first global approval. Drugs. 2014;74:1829-36. 